\title{
Depinning and dynamics of imbibition fronts in paper under increasing ambient humidity
}

\author{
Alexander S. Balankin, ${ }^{1}$ H. Zapata López, ${ }^{1}$ E. Pineda León, ${ }^{1}$ D. Morales Matamoros,${ }^{2}$ L. Morales Ruiz, ${ }^{1}$ \\ Dan Silva López, ${ }^{1}$ and M. A. Rodríguez ${ }^{3}$ \\ ${ }^{1}$ Grupo “Mecánica Fractal," Instituto Politécnico Nacional, México D.F., Mexico 07738 \\ ${ }^{2}$ Instituto Mexicano del Petróleo (IMP), México D.F., Mexico 07730 \\ ${ }^{3}$ Instituto de Física de Cantabria (IFCA), CSIC-UC, E-39005 Santander, Spain
}

(Received 12 October 2012; revised manuscript received 14 December 2012; published 15 January 2013)

\begin{abstract}
We study the effects of ambient air humidity on the dynamics of imbibition in a paper. We observed that a quick increase of ambient air humidity leads to depinning and non-Washburn motion of wetting fronts. Specifically, we found that after depinning the wetting front moves with decreasing velocity $v \propto\left(h_{p} / h_{D}\right)^{\gamma}$, where $h_{D}$ is the front elevation with respect to its pinned position at lower humidity $h_{p}$, while $\gamma \cong 1 / 3$. The spatiotemporal maps of depinned front activity are established. The front motion is controlled by the dynamics of local avalanches directed at $30^{\circ}$ to the balk flow direction. Although the roughness of the pinned wetting front is self-affine and the avalanche size distribution displays a power-law asymptotic, the roughness of the moving front becomes multiaffine a few minutes after depinning.
\end{abstract}

PACS number(s): 05.70.Ln, 47.56.+r, 47.55.nb, 45.70.Ht

Fluid imbibition in porous media plays an important role in many natural phenomena and technological processes, ranging from oil recovery to printing [1]. Besides, imbibition phenomena bring forward challenging questions from a fundamental point of view, in particular in statistical physics as nonequilibrium dynamic processes [1,2]. Starting from classical works [3], paper sheets were widely used as porous media in studies of imbibition and related phenomena [4-11]. An advantage of paper wetting experiments is that the wetting front configurations are easily observable in situ, since the associated time and spatial scales are easily accessible in the laboratory studies. Although extensive experimental and theoretical research has been performed in the past decade to understand the dynamics and pinning of imbibition fronts in porous media [7,12-19], several aspects of related phenomena remain unknown or poorly understood.

One of the open problems concerns the effect of ambient conditions (air humidity and temperature) on the fluid imbibition, associated with such phenomena as prewetting and saturation of porous media, moisture swelling and water evaporation, precursor film propagation and fingering, among others (see Refs. [5,8,20,21], and references therein). In this Brief Report we describe some noteworthy features of wetting front dynamics observed in paper imbibition experiments after a quick increase in ambient air humidity.

To control the air humidity, all experiments were performed in a climate box with controlled temperature $\left(25 \pm 5^{\circ} \mathrm{C}\right)$ and relative humidity $(\mathrm{RH} \pm 5 \%)$ of air. Sheets $\left(500 \times 200 \mathrm{~mm}^{2}\right)$ of filter paper [22] were clipped to a ring stand and submerged into a reservoir filled with black Chinese-ink suspension [23]. The reservoir size was large enough to assure the free surface of ink suspension at a constant level $z=0$ during the experiment. All height measurements were made taking into account this reference level. Once the sheet bottom is submerged to a depth of about $5 \mathrm{~mm}$ below the reference level in the reservoir, the ink impregnates the paper, forming a rough interface between the wet and dry regions which rises along the paper due to the capillary action. The position and configuration of the wetting front were monitored by taking high resolution (6.5 megapixels) photographs every $20 \mathrm{~s}$ during the experiment. Further, all snapshots were digitized and plotted as single-valued functions $z(x, t)$ (for more details see Ref. [8]). The mean height of the wetting front at time $t$ is defined as $h(t)=\langle z(x, t)\rangle$, where the brackets denote the average over the sheet width, while the front global width is defined as $W(t)=\max _{x} z(x, t)-\min _{x} z(x, t)$.

It is pertinent to note that the effect of ambient air humidity on dynamics and pinning of wetting fronts in the Filtro paper was earlier studied in Ref. [8] in which two different scenarios of imbibition dynamics (associated with low and high evaporation rates) were observed. In both cases, imbibition fronts are stopped by pinning forces at the height $h_{p}$ much lower than the equilibrium height of imbibition $h_{e}=P_{c} / \rho g$ determined by the balance of capillary $\left(P_{c}\right)$ and gravitational $(\rho g h)$ forces. Notice that although in some experiments with papers the Washburn regime $h \propto t^{1 / 2}$ was observed within bounded intervals of time scale (see Refs. [1,8]), in most of the paper imbibition experiments it was found that the wetting front rises as

$$
h \propto t^{\delta}
$$

with the scaling exponent in the range of $0.3 \leqslant \delta<0.5$ (see, for review, Ref. [24]), whereas the global front width increases as $W \propto t^{\alpha}$, where $\alpha$ is the global growth exponent [14,19]. As the imbibition front advances, the net loss of water mass per unit time due to evaporation increases, because of an increase in the wetted area. Accordingly, the pinning height $h_{p}$ depends on the evaporation rate which decreases with the increase of ambient air humidity [8].

In this work, all imbibition experiments were started at low ambient humidity $\left(\mathrm{RH}_{1}=35 \pm 5 \%\right)$. Further, after the wetting front is completely pinned at the height $h_{p}$ of about $120 \pm 20 \mathrm{~mm}$ and no changes in either height or shape of the interface between the wet and dry regions were observed during $10 \mathrm{~min}$, the water vapor was injected in the climatic box [25]. In this way, we found that a quick increase of air humidly up to $\mathrm{RH}_{2} \geqslant 65 \%$ leads to depinning of the wetting front. Figure 1 shows the digitized snapshots of the depinned front moving along the sheet. We found that the height of the depinned wetting front $\left[h_{p}<h(t)<h_{M}<300 \mathrm{~mm}<h_{e} \approx 500 \mathrm{~mm}\right]$ 


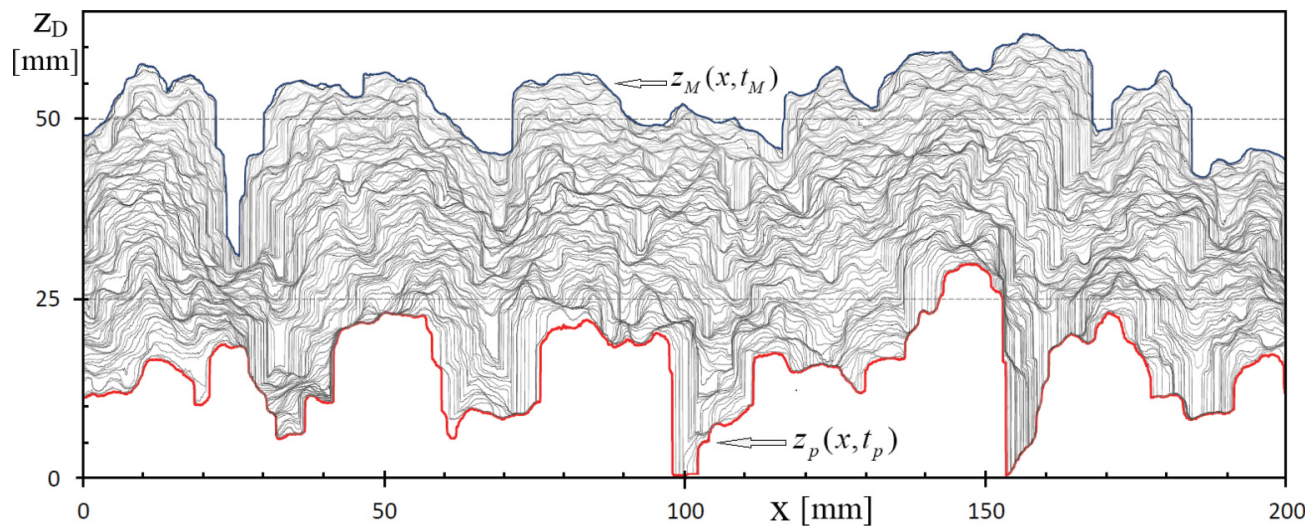

FIG. 1. (Color online) Time evolution of the depinned wetting front $z_{D}(x, t)=z(x, t)-\min _{x} z_{p}\left(x, t_{p}\right)$ after vapor injection (notice that, for clarity, the front configurations are shown every $40 \mathrm{~s}$, instead of the interval of $20 \mathrm{~s}$ used in experiments). $z_{p}\left(x, t_{p}\right)$ represents the pinned configuration of the wetting front in experiment at $\mathrm{RH}_{1}=30 \pm 1 \%$ and $T=23 \pm 0.7^{\circ} \mathrm{C}$. Vapor injection (during $30 \mathrm{~s}$ ) leads to an increase of air humidity $\mathrm{RH}_{2}=75 \pm 2 \%$ and temperature $T=28 \pm 2{ }^{\circ} \mathrm{C} . z_{M}\left(x, t_{M}\right)$ represents the configuration of the front pinned at high air humidity after $2 \mathrm{~h}$.
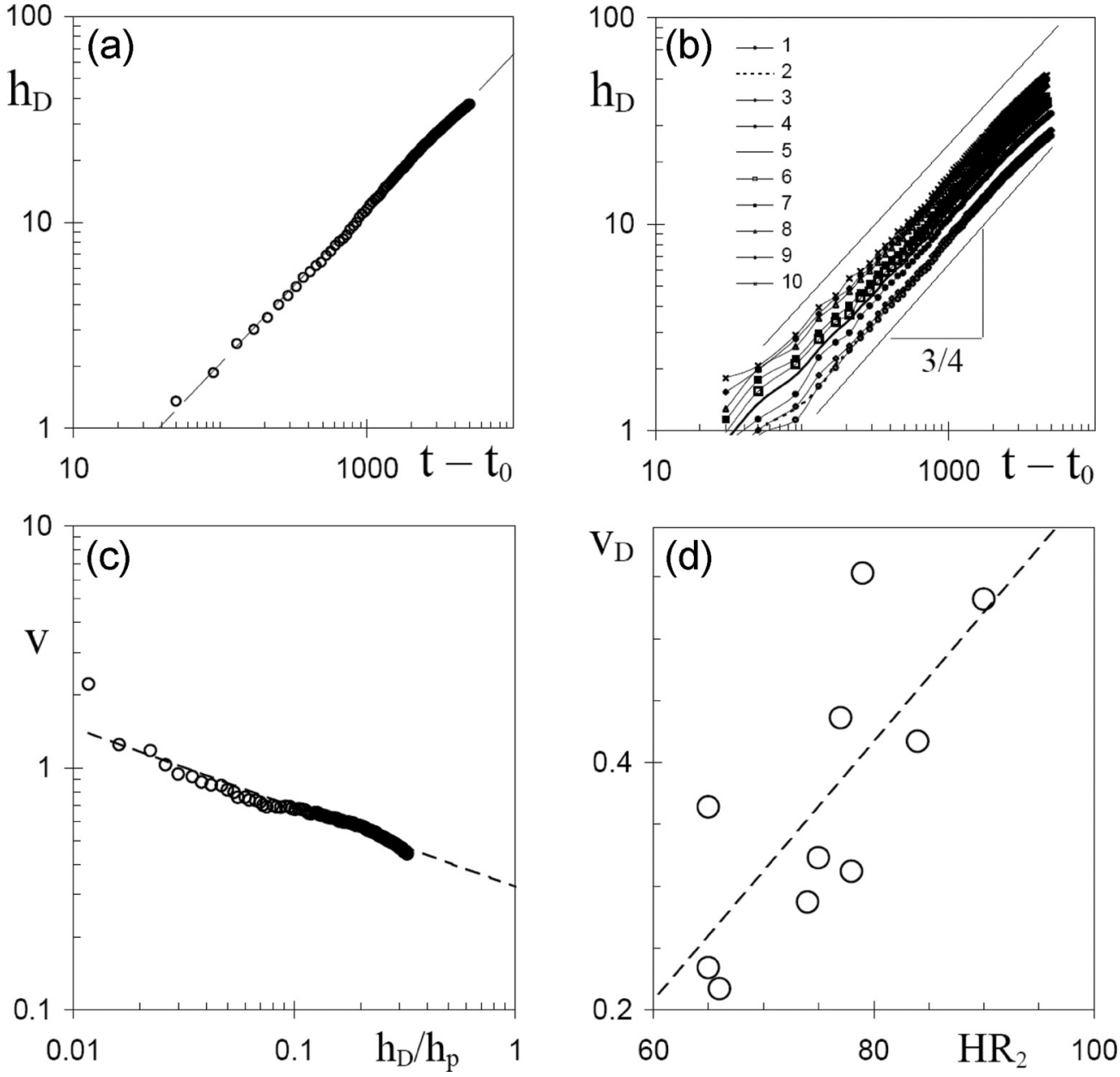

FIG. 2. The log-log graphs of $h_{D}(t)$ in millimeters versus $\left(t-t_{0}\right)$ in seconds for (a) experimental data shown in Fig. 1; straight line represents the best fit $\left(R^{2}=0.99\right)$ with $t_{0}=70 \mathrm{~s}$ and $\delta_{D}=0.752$ for $t \geqslant t_{0}+\tau_{0} \geqslant 120 \mathrm{~s}$ and (b) for ten experiments performed at different air humidity; curves from bottom to top correspond to $\mathrm{RH}_{2}=65 \%, 65 \%, 66 \%, 74 \%, 75 \%, 77 \%, 78 \%, 79 \%$, 84\%, and $90 \%$; straight lines are a guide for the eye only. The graphs of (c) front velocity $v$ (in $\mathrm{mm} / \mathrm{min}$.) versus the dimensionless ratio $h_{D} / h_{p}$ for experiment shown in Fig. 1 ; straight line—data fitting by Eq. (3) with $\beta=\left(1-\delta_{D}\right) / \delta_{D}=1 / 3$. (d) Fitting parameter $v_{D}$ (in $\mathrm{mm} / \mathrm{min}$.) versus $\mathrm{RH}_{2}$ (in percent); straight line shows the tendency. 

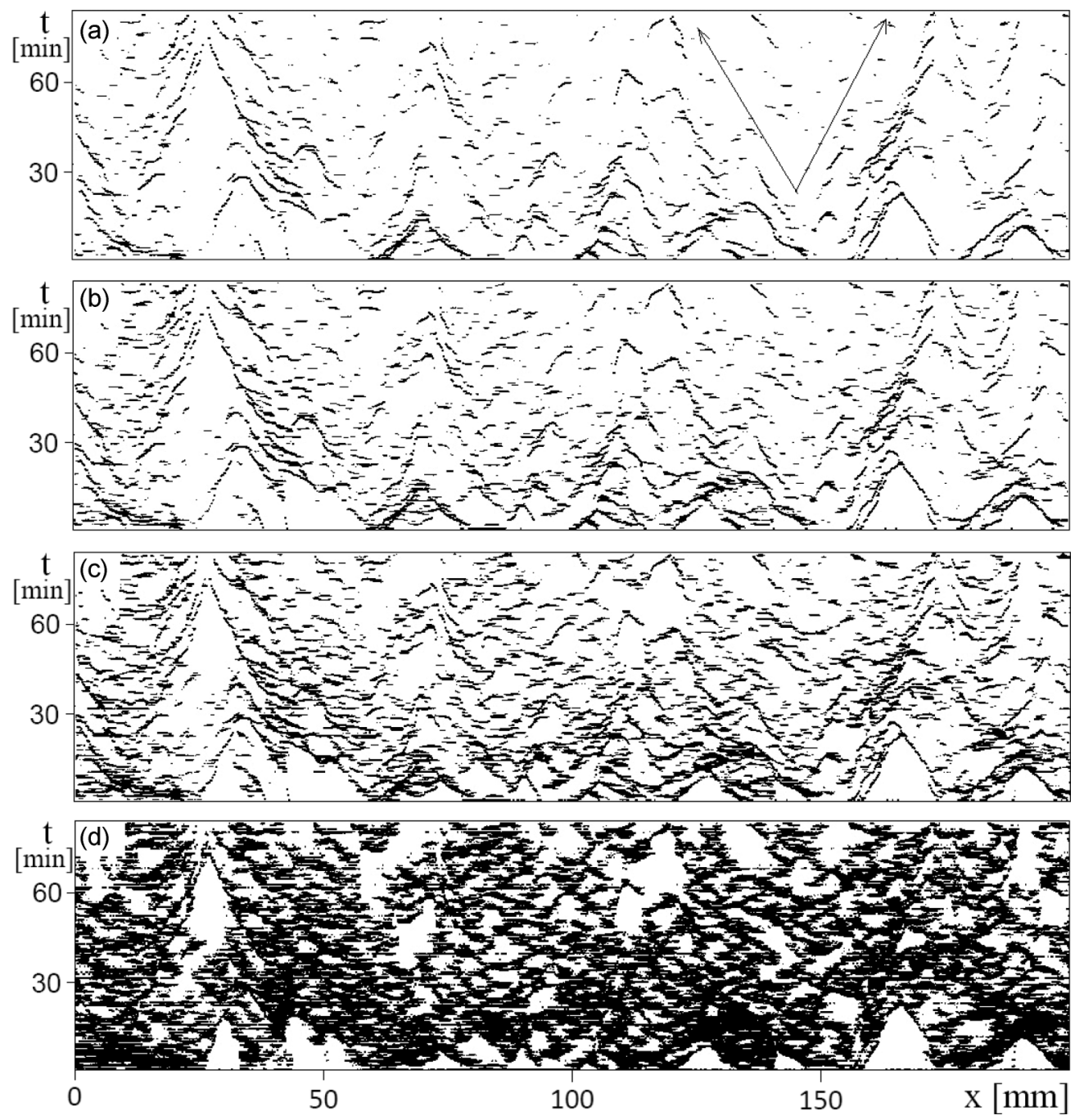

FIG. 3. Spatiotemporal distribution of imbibition activity obtained from the evolution of the wetting front after fast increase in ambient air humidity (for experiment shown in Fig. 1) at different thresholds $\Delta_{\text {th }}=$ (a) 7, (b) 5, (c) 3, and (d) 1 pixel $=0.063 \mathrm{~mm}$.

increases in time as

$$
h_{D}=h(t)-h_{p}=a\left(t-t_{0}\right)^{\delta_{D}} \quad \text { for } t \geqslant t_{0}+\tau_{0},
$$

taking $t=0$ at the beginning of vapor injection [25], where the scaling exponent $\delta_{D}=0.75 \pm 0.4$ [26] [see Fig. 2(a)] is found to be almost independent on the experiment conditions [see Fig. 2(b)], while $t_{0}, \tau_{0}$, and $a$ are fitting parameters [27]. Notice that, although Eq. (2) resembles Eq. (1) of the wetting front climb at constant ambient humidity, the depinned front rises faster $\left(\delta_{D}=0.75 \pm 0.4\right)$ than in the Washburn regime $(\delta=0.5)$, in contrast to the slower rise $(\delta=0.41 \pm 0.05)$ at the initial stage $h<h_{p}\left(\mathrm{RH}_{1}\right)$ [28]. Furthermore, experimental data suggest that at the stage of $\mathrm{RH}_{2}>\mathrm{RH}_{1}$ the velocity of the depinned wetting front obeys the following equation of motion:

$$
v=\frac{\partial h}{\partial t}=v_{D}\left(\frac{h_{p}}{h_{D}}\right)^{\gamma} \text { for } \quad h_{D}>h_{D}\left(\tau_{0}\right)
$$

with the scaling exponent $0<\gamma=\left(1-\delta_{D}\right) / \delta_{D}=0.33 \pm$ $0.02 \cong 1 / 3$ [see Fig. 2(c)], while $h<h_{M}<h_{e}$ and the fitting parameter $v_{D}=h_{p} \delta_{D}\left(a / h_{p}\right)^{1 / \delta_{D}}$ is an increasing function of $\mathrm{RH}_{2}$ [see Fig. 2(d)].
In order to characterize the wetting front dynamics, we analyze the spatiotemporal distributions of wetting activity after depinning. Figure 3 shows typical maps of wetting activity in which the black zones represent front advances

$$
\Delta z_{D}(x, t)=z_{D}(x, t)-z_{D}(x, t-20 \mathrm{~s}) \geqslant \Delta_{\mathrm{th}},
$$

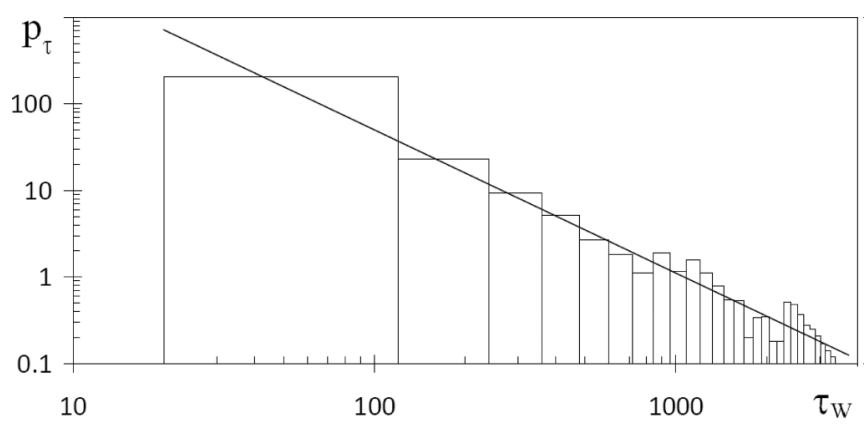

FIG. 4. Log-log graph of probability density $p_{\tau}$ (in $1 / \mathrm{s}$ ) of waiting times $\tau_{W}$ (in seconds). Bins-experimental data averaged over ten experiments at different air humidity in the range of $65 \% \leqslant \mathrm{RH}_{2} \leqslant$ 90\%; straight line-power-law fit $p_{\tau} \propto \tau_{W}^{-\alpha_{\tau}}$ with $\alpha_{\tau}=1.65$. 


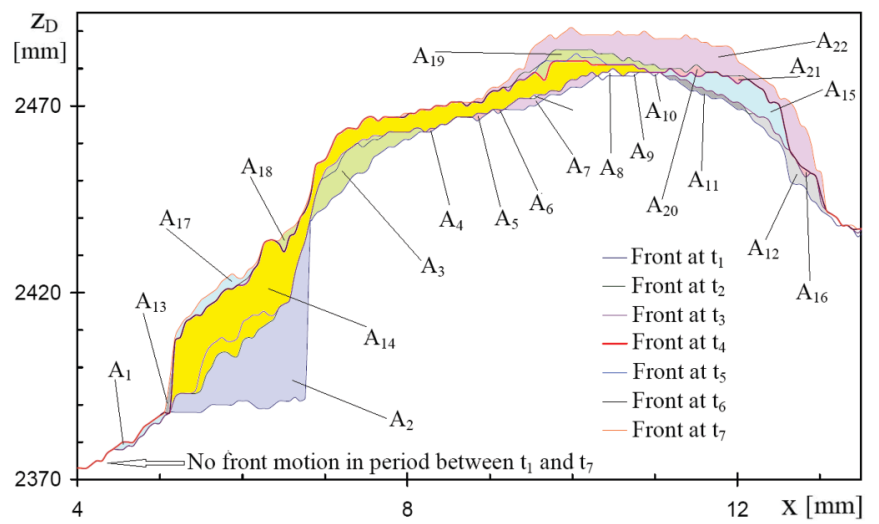

FIG. 5. (Color online) Time evolution of the fragment of the depinned wetting front in the period between $t_{1}=82$ and $t_{7}=84 \mathrm{~min}$. after vapor injection. Notice the local superpositions of front curves at different $t_{i} . A_{j}$ denotes the local avalanches defined as continuously wetted areas between front configurations. The global avalanche can be defined as the set of local avalanches $A_{j}$ with $1 \leqslant j \leqslant 12$.

where $\Delta_{\text {th }}$ is the threshold value. Hence, the maps of Fig. 3 can also be interpreted as the spatiotemporal distributions of local velocities, such that the black zones correspond to local velocities $v_{l}(x, t)=\Delta z_{D} / 20 \geqslant v_{\text {th }}=\Delta_{\text {th }} /(20 \mathrm{~s})$. One can see that the depinned front moves due to localized advances with a broad distribution of local velocities [compare maps (a)-(d) in Fig. 3], rather than by the continuous capillary flow. We found that the distribution $p_{\tau}$ of waiting times $\tau_{W}$ between subsequent local advances $\Delta z_{D}(x, t) \geqslant 1$ pixel in the flow direction [see Fig. 3(d)] obeys power-law behavior $p_{\tau} \propto \tau_{W}^{-\alpha_{\tau}}$ with the scaling exponent $\alpha_{\tau}=1.65 \pm 0.5$ (see Fig. 4).

Furthermore, we recognized that the local advances form clusters of spatiotemporal wetting activity directed at $30^{\circ}$ to the balk flow direction (see Fig. 3). We also noted that this feature was observed in the numerical simulation of wetting front activity near the depinning transition (see Ref. [16]). In addition, we found that the deviation of local flow from the main flow direction leads to the change in the $x$ coordinate of the maximum elevation $z_{m}(t)=\max _{x} z_{D}(x, t)$ (see Fig. 1). Consequently, $z_{m}(t)$ displays a Devil's-staircase-like behavior with the Hurst exponent $\delta_{m}=0.67 \pm 0.08$ (determined by the power-spectrum method with the help of BENOIT 1.3 software [29]), qualitatively similar to the behavior of $z_{m}(t)$ at low humidity reported in Ref. [6]. However, the value of Hurst exponent $\delta_{m}=0.67 \pm 0.08$ is larger than $\delta_{m}=0.26 \pm 0.01$ earlier found in imbibition experiments with the same paper but before the pinning at low ambient humidity (see Ref. [6]).

Intermittent spatiotemporal activity (see Fig. 3) necessarily leads to the formation of a set of local avalanches which are disconnected in the space and time (see Ref. [30]). In addition, one can define the global avalanches associated with the intermittent temporal dynamics of the whole front. Near to the pinning-depinning transition it is expected that the size distributions of local and global avalanches both display power-law asymptotics, but with different scaling exponents.
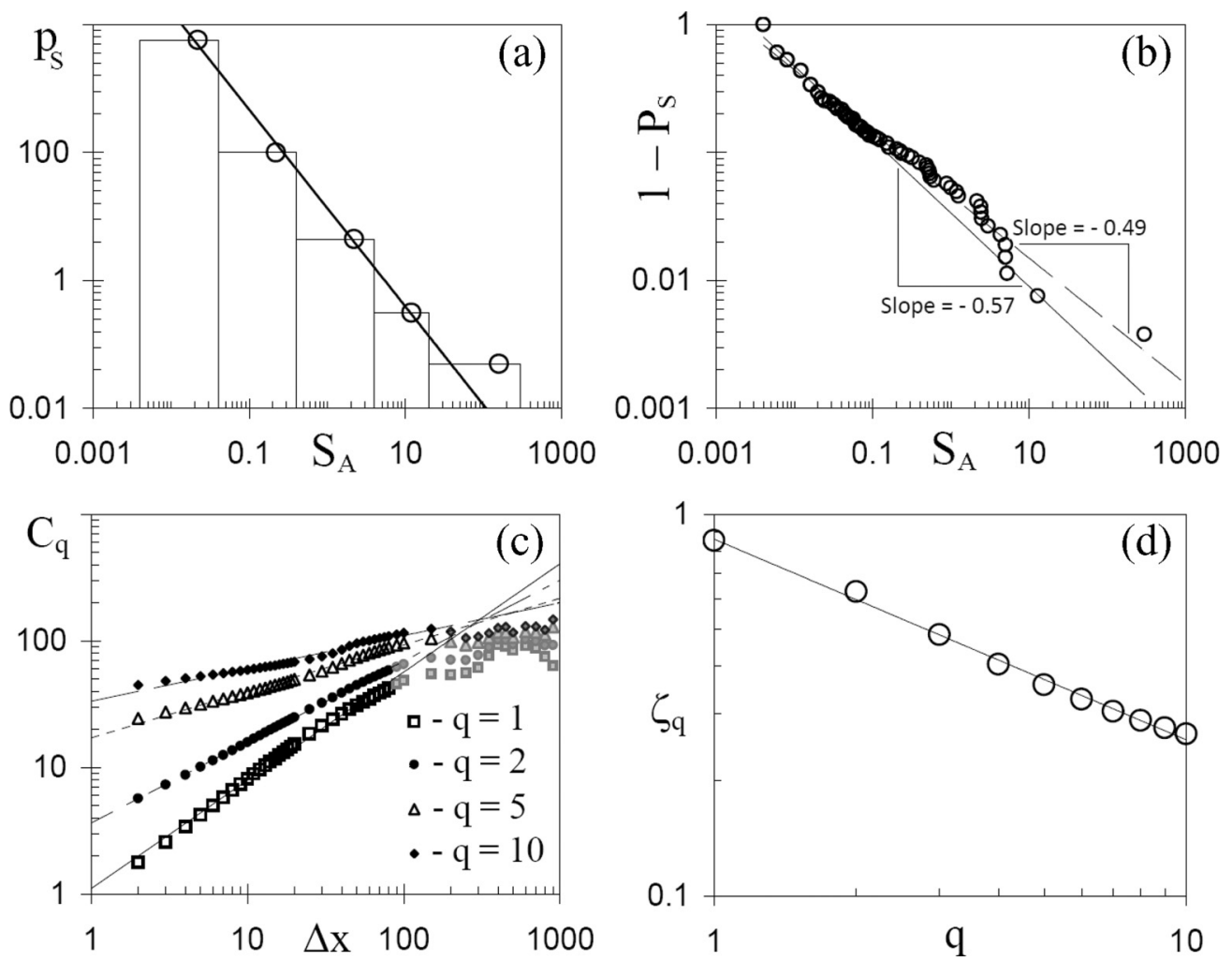

FIG. 6. (a) Probability density distribution $p_{S}$ (in $1 / \mathrm{mm}^{2}$ ) of local avalanche areas $S_{A}$ (in $\mathrm{mm}^{2}$ ): bins-experimental data averaged over ten experiments at different air humidity; straight line-power-law fit $p_{S} \propto S_{A}^{-\alpha_{A}}$ with $\alpha_{A}=1.537$ and (b) the corresponding survival function $1-P_{S}$, where $P_{S}=N\left(S_{A}\right) / N_{\text {Total }}$ is the dimensionless cumulative distribution, while $N_{\text {Total }}=264$. (c) Log-log plot of the $q$-order height-height correlation functions $C_{q}$ versus window size $\Delta x$ for different $q$ and (d) spectrum of multiaffine exponents $\zeta_{q}$ versus $q$. 
In the context of our experiments, the local avalanches can be defined as continuously wetted areas between front configurations $z\left(x, h_{D}\right)$ at different mean heights $h_{D}(t)$ (see Fig. 5). A set of spatially disconnected local avalanches form the global avalanches (see Ref. [30]), while the total area of avalanches is equal to the total area wetted after front depinning (see Fig. 1). We found that the probability distribution of avalanche areas $S_{A}$ displays a power-law asymptotic with exponent $\alpha_{A}=1.54 \pm 0.05$ [see Figs. 6(a) and 6(b)]. We noted that this value is consistent with the values of local avalanche size distribution exponents found in numerical simulations of fluid imbibition near the depinning transition $\left(\alpha_{A}=1.54\right)$ [16] and planar crack front propagation $\left(\alpha_{A}=1.5\right)$ [30].

In this context, notice that the authors of [30] have claimed that their results are applicable also to other systems with long-range interactions near a nonequilibrium phase transition. Furthermore, the authors of Ref. [30] have shown that the power-law exponent of the global avalanche size distribution is related to $\alpha_{A}$ as $\alpha_{G A}=2 \alpha_{A}-1$. Although we found that our observations are qualitatively consistent with this relation, we were not able to confirm it quantitatively, because of the larger scatter in experimental data [31]. Nonetheless, some features of wetting activity observed in this work [see Figs. 3, 5(a), and 5(b)] can be attributed to the effects of long-range interactions in the system.

In addition, the model suggested in [30] predicts a self-affine roughness of the moving front. However, we found that a few minutes after depinning, the moving wetting front displays a multiaffine roughness [32] characterized by a broad spectrum of scaling exponents $\zeta_{q}=0.86 q^{0.526}$ [see Figs. 6(c) and 6(d)] defined from the scaling behavior of $q$-order height-height correlation functions $C_{q}(\Delta x)=\left(L^{-1} \sum_{x=0}^{L}|z(x)-z(x+\Delta x)|^{q}\right)^{1 / q} \quad$ (see, for details, Ref. [33]). Furthermore, in contrast to the increase of global front width $W \propto t^{\alpha}$ expected in the case of kinetic roughening and always observed in paper imbibition experiments at a constant humidity [4-10], we found that the global width of the wetting front moving in regime (3) tends to decrease in time (see Fig. 1). The reasons of observed front roughening dynamics require further analysis.

Summarizing, we found that a quick increase in ambient air humidity leads to depinning and decelerating non-Washburn motion of initially pinned wetting fronts in paper imbibition experiments. The front depinning under increasing ambient humidity can be attributed to the decay of the evaporation rate. The motion of the depinned front is controlled by spatially disconnected local avalanches directed at $30^{\circ}$ to the bulk imbibition direction. The distributions of waiting times and local avalanche sizes display power-law asymptotics, whereas the front roughness displays multiaffine scaling with a broad spectrum of scaling exponents and the front global width tends to decrease as the front advances after depinning. Nonetheless with the complexity of the wetting dynamics under increasing ambient humidity, we found that a non-Washburn motion of the depinned wetting front obeys the simple empirical equation of motion (3). We expect that our findings will stimulate further experimental and theoretical research of imbibition phenomena and depinning dynamics under decreasing pinning force.

This work was supported by the PEMEX under the research grants SENER-CONACYT No. 143927 and No. 116458.
[1] M. Alava, M. Dubé, and M. Rost, Adv. Phys. 53, 83 (2004); D. Bonn, J. Eggers, J. Indekeu, J. Meunier, and E. Rolley, Rev. Mod. Phys. 81, 046311 (2009).

[2] Imbibition is a process by which a wetting fluid is drawn into a capillary or porous medium and displaces a lesser wetting fluid (or gas) occupying the space. Spontaneous imbibition takes place when the invading fluid does so under the sole influence of capillary forces, with no external pressure. Imbibition in porous media gives rise to many interesting phenomena, including kinetic roughening of moving wetting fronts and their pinning, which have received a considerable amount of interest [1].

[3] J. M. Bell and F. K. Cameron, J. Phys. Chem. 10, 658 (1906); E. Lucas, Kolloid-Z. 23, 15 (1918); E. W. Washburn, Phys. Rev. 17, 273 (1921)

[4] S. V. Buldyrev, A.-L. Barabási, S. Havlin, J. Kertész, H. E. Stanley, and H. S. Xênias, Physica A 191, 220 (1992); S. V. Buldyrev, A.-L. Barabási, F. Caserta, S. Havlin, H. E. Stanley, and T. Vicsek, Phys. Rev. A 45, R8313 (1992); L. A. N. Amaral, A.-L. Barabási, S. V. Buldyrev, S. T. Harrington, S. Havlin, R. Sadr-Lahijany, and H. E. Stanley, Phys. Rev. E 51, 4655 (1995); V. K. Horváth and H. E. Stanley, ibid. 52, 5166 (1995); P. B. Sunil Kumar and D. Jana, Physica A 224, 199 (1996); A. Takahashi, M. Häggkvist, and T.-Q. Li,
Phys. Rev. E 56, 2035 (1997); M. Dubé, B. Chabot, C. Daneault, and M. Alava, Pulp Pap. 106, 24 (2005).

[5] L. A. N. Amaral, A.-L. Barabási, S. V. Buldyrev, S. Havlin, and H. E. Stanley, Phys. Rev. Lett. 72, 641 (1994).

[6] A. S. Balankin, A. Bravo-Ortega, and D. Morales, Philos. Mag. Lett. 80, 503 (2000).

[7] A. S. Balankin, O. Susarrey, and J. M. Gonzáles, Phys. Rev. Lett. 90, 096101 (2003).

[8] A. S. Balankin, R. Paredes, O. Susarrey, D. Morales, and F. Castrejon, Phys. Rev. Lett. 96, 056101 (2006).

[9] E. N. de Azevedo, L. R. Alme, M. Engelsberg, J. O. Fossum, and P. Dommersnes, Phys. Rev. E 78, 066317 (2008).

[10] A. M. Miranda, I. L. Menezes-Sobrinho, and M. S. Couto, Phys. Rev. Lett. 104, 086101 (2010).

[11] J. Kim, M.-W. Moon, K.-R. Lee, L. Mahadevan, and H.-Y. Kim, Phys. Rev. Lett. 107, 264501 (2011).

[12] D. Geromichalos, F. Mugele, and St. Herminghaus, Phys. Rev. Lett. 89, 104503 (2002); S. Supple and N. Quirke, ibid. 90, 214501 (2003); C.-H. Lam, ibid. 92, 254503 (2004); J. Soriano, A. Mercier, R. Planet, A. Hernández-Machado, M. A. Rodríguez, and J. Ortín, ibid. 95, 1045015 (2005); S. Gruener and P. Huber, ibid. 103, 174501 (2009); R. Planet, St. Santucci, and J. Ortín, ibid. 102, 094502 (2009); P. Gaillard, Y. Saito, 
and O. Pierre-Louis, ibid. 106, 195501 (2011); A. Marchand, S. Das, J. H. Snoeijer, and B. Andreotti, ibid. 108, 094301 (2012); L. Cueto-Felgueroso and R. Juanes, ibid. 108, 144502 (2012); A. O. Parry, C. Rascón, E. A. G. Jamie, and D. G. A. L. Aarts, ibid. 108, 246101 (2012).

[13] B. Lavi and A. Marmur, Colloids Surf., A 282-283, 263 (2006); C. U. Hatiboglu and T. Babadagli, Phys. Rev. E 77, 066311 (2008); N. Fries and M. Dreyer, J. Colloid Interface Sci. 338, 514 (2009); K. Li, J. Contam. Hydrol. 111, 13 (2010); B. Amaziane, J. P. Milisic, M. Panfilov, and L. Pankratov, Phys. Rev. E 85, 016304 (2012); F. Doster, O. Hönig, and R. Hilfer, ibid. 86, 016317 (2012).

[14] M. Pradas and A. Hernández-Machado, Phys. Rev. E 74, 041608 (2006); R. Planet, M. Pradas, A. Hernández-Machado, and J. Ortín, ibid. 77, 056312 (2007); M. Dubé, C. Daneault, V. Vuorinen, M. Alava, and M. Rost, ibid. 56, 15 (2007); M. Pradas, J. M. López, and A. Hernández-Machado, ibid. 76, 010102(R) (2007); M. Pradas, A. Hernández-Machado, and M. A. Rodríguez, ibid. 77, 056305 (2008); R. Planet, S. Santucci, and J. Ortín, J. Contam. Hydrol. 120-121, 157 (2011).

[15] J. Cai, B. Yu, M. Zou, and M. Mei, Chem. Eng. Sci. 65, 5178 (2010); J. Cai, B. Yu, M. Zou, and L. Luo, Energy Fuels 24, 1860 (2010).

[16] M. Pradas, J. M. López, and A. Hernández-Machado, Phys. Rev. E 80, 050101(R) (2009).

[17] J. M. López, M. Pradas, and A. Hernández-Machado, Phys. Rev. E 82, 031127 (2010).

[18] N. Obara and K. Okumura, Phys. Rev. E 86, 020601(R) (2012).

[19] A. S. Balankin, E. García Otamendi, D. Samayoa, J. Patiño, and M. A. Rodríguez, Phys. Rev. E 85, 036313 (2012).

[20] J. Ghassemzadeh, M. Hashemi, L. Sartor, and M. Sahimi, AIChE J. 47, 519 (2001); Y. X. Wang and G. P. Peterson, J. Thermophys. Heat Transfer 17, 145 (2003); A. Brú and J. M. Pastor, Geoderma 134, 295 (2006); O. Chapuis and M. Prat, Phys. Rev. E 75, 046311 (2007); N. Shokri and M. Sahimi, ibid. 86, 066312 (2012); A. G. Yiotis, D. Salin, E. S. Tajer, and Y. C. Yortsos, ibid. 86, 026310 (2012).
[21] M. Dubé, M. Rost, and M. Alava, Eur. Phys. J. B 15, 691 (2000); M. Dubé, S. Majaniemi, M. Rost, M. J. Alava, K. R. Elder, and T. Ala-Nissila, Phys. Rev. E 64, 051605 (2001).

[22] Filter paper with open porosity is a highly porous material and contains as much as $73 \%$ air. The normal moisture content of paper is a few percent. Some structural and physical properties of this paper are reported in [7].

[23] The black Chinese ink is a dilute mixture of colloidal black carbon particles and water. In this work we used the $15 \%$ ink solution in water.

[24] J. Cai and B. Yu, Transp. Porous Media 89, 251 (2011).

[25] The duration of vapor injection was less than 1 min., while the air humidity and temperature were stabilized in a few minutes.

[26] Error bars correspond to the standard deviations of $\delta$ obtained in ten experiments with different $\mathrm{RH}_{2}$.

[27] We found that $t_{0}$ and $\tau_{0}$ vary in the interval $20 \leqslant \tau_{0}<t_{0} \leqslant 240 \mathrm{~s}$ for experiments with $65 \% \leqslant \mathrm{RH}_{2} \leqslant 90 \%$. We also noted that the fitting parameter $a$ and the maximum height achieved by the wetting front after depinning $h_{M}$ both increase as $\mathrm{RH}_{2}$ increases [see Fig. 2(b)].

[28] In this context we noted that most models devoted to explain a non-Washburn behavior of imbibition in porous media predict the scaling behavior (1) with $\delta<0.5$ (see Refs. [15,24], and references therein).

[29] BENOIT 1.3, http://www.trusoft-international.com; W. Seffens, Science 285, 1228 (1999).

[30] L. Laurson, S. Santucci, and S. Zapperi, Phys. Rev. E 81, 046116 (2010).

[31] The number of global avalanches per experiment is less than 20. The average between ten experiments at different air humidity suggests that $\alpha_{G A}=1.7 \pm 0.5$. This value is consistent with the theoretical relation $\alpha_{G A}=2 \alpha_{A}-1=2.1 \pm 0.1$ derived in Ref. [30].

[32] The roughness of pinned fronts is found to be self-affine with the roughness (Hurst) exponent $\zeta=0.78 \pm 0.01$ (see also Refs. [6-8]).

[33] A. S. Balankin and D. Morales Matamoros, Phys. Rev. E 71, 056102 (2005). 